\title{
Salvia miltiorrhiza prevents deep vein thrombosis via antioxidative effects in endothelial cells
}

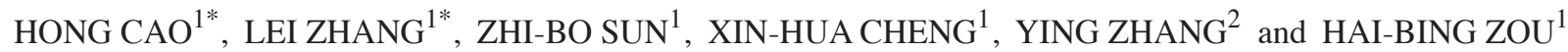 \\ ${ }^{1}$ Department of Orthopedic Surgery and ${ }^{2}$ Reproductive Medicine Center, \\ Renmin Hospital, Hubei University of Medicine, Shiyan, Hubei 442000, P.R. China
}

Received March 3, 2014; Accepted December 9, 2014

DOI: $10.3892 / \mathrm{mmr} .2015 .3153$

\begin{abstract}
Deep vein thrombosis (DVT) is a common clinical problem, which represents a significant clinical and economic burden. The present study investigated whether Salvia miltiorrhiza (S. miltiorrhiza) could prevent DVT. A total of 30 rabbits were randomly divided into three groups $(n=10$ per group): The control, model and Salvia groups. A ligation model was used, where the femoral veins of rabbits were exposed and ligated. Measurements of coagulation function, blood rheological parameters, antioxidative function and effects on endothelial cells were conducted. Treatment with S. miltiorrhiza one week prior to generation of the ligation model did not affect the coagulation function much, except to increase the prothrombin time. There was a statistically significant difference $(\mathrm{P}<0.05)$ in whole blood viscosity $(1 / \mathrm{s}$, $5 / \mathrm{s}, 30 / \mathrm{s})$ on the third and seventh days (1/s, 5/s, 30/s and 200/s) following generation of the model. S. miltiorrhiza exhibited promising antioxidative effects, as demonstrated by a significant decrease in malondialdehyde content $(\mathrm{P}<0.05)$, and an increase in the activities of superoxide dismutase $(\mathrm{P}<0.05)$, as compared with the model group. S. miltiorrhiza was also shown to protect the vascular endothelial cells, as compared with the model group. These results suggest that $S$. miltiorrhiza may have potential applications for the treatment of DVT.
\end{abstract}

\section{Introduction}

Deep vein thrombosis (DVT) is a common clinical problem that affects $>250,000$ individuals in the USA and 25,000 individuals in Canada annually (1). DVT in the lower

Correspondence to: Hai-Bing Zou, Department of Orthopedic Surgery, Renmin Hospital, Hubei University of Medicine, Chao Yang Road, 39th Street, Shiyan, Hubei 442000, P.R. China

E-mail: 33556511@qq.com

${ }^{*}$ Contributed equally

Key words: deep vein thrombosis, Salvia miltiorrhiza, antioxidative function, endothelial cells extremities is characterized by initial swelling and lower limb pain symptoms, which may be severe. DVT is also a well-recognized cause of pulmonary embolism (PE), which is a potentially life-threatening complication $(2,3)$, for which treatment is necessary. Traditionally, the initial treatment for DVT involves anticoagulation using low-molecular-weight heparin $(4,5)$, followed by long term therapy with vitamin $\mathrm{K}$ antagonists, such as warfarin (6). Since the outcome of these treatments in some cases is disappointing $(7,8)$, more effective approaches aimed at reducing DVT have garnered interest in recent years.

Salvia miltiorrhiza is one of the most versatile Chinese herbal drugs, which has been used for hundreds of years to treat numerous ailments (9). S. miltiorrhiza is considered to be highly effective in activating circulation, and dispersing stasis or sludging of blood (10). In recent years, S. miltiorrhiza has been widely used to treat cardiovascular diseases (11). The present study investigated whether S. miltiorrhiza could prevent DVT, using a rabbit ligation model. Furthermore, the underlying mechanism of the protective effects of S. miltiorrhiza on vascular endothelial cells was examined.

\section{Materials and methods}

Materials. S. miltiorrhiza was donated by the Pharmacy of the Hubei University of Medicine (Shiyan, China) (no. Z51021303). These were extractions of roots, containing $1.5 \mathrm{~g} / \mathrm{ml}$. Malondialdehyde (MDA) and Superoxide Dismutase (SOD) Detection kits were obtained from Nanjing Jiancheng Institute of Biotechnology (Nanjing, China). A total of 30 male Japanese white rabbits, weighing 1.8-2.2 kg, were obtained from the Animal Care Facility at the Hubei Animal Center (Wuhan, China) (no. SYXK 2004-0021). All the rabbits had ad libitum access to food and water and were under a standard $12 \mathrm{~h}$ light/12 h dark cycle. All experiments performed were in accordance with animal ethics standards. Prior to the experiment, the rabbits were maintained for several days, to observe their health. The rabbits were randomly divided into three groups ( $\mathrm{n}=10$ per group): The control, model and Salvia groups. All procedures were approved by the Animal Research Ethics Board at Hubei University of Medicine.

Modeling method. All surgical manipulations were conducted under aseptic conditions, and the rabbits were anesthetized 

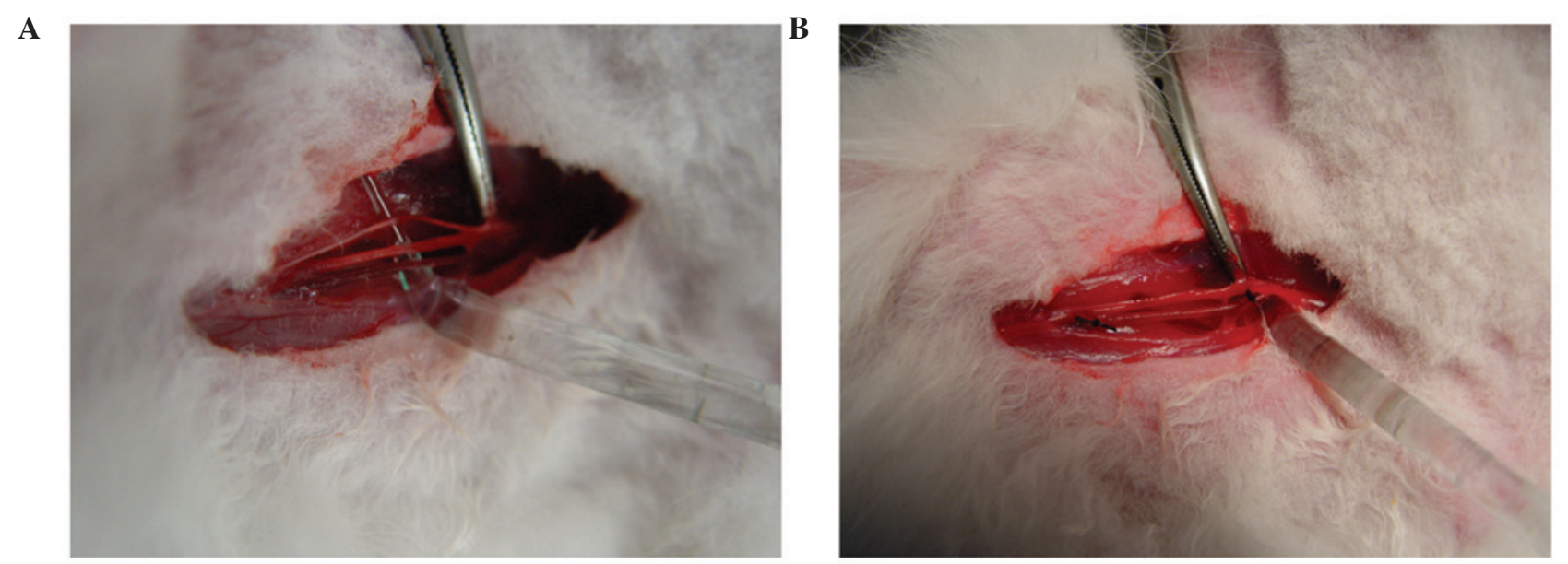

Figure 1. Following anesthetization, the femoral vein, artery and nerve of the rabbits were exposed from the surrounding tissue. (A) From left to right are the femoral nerve, artery and vein. (B) Each end of the femoral vein was completely ligated using 2-0 silk thread.

with $10 \%$ chloral hydrate (Weihai Wego Medical Systems Co., Ltd, Weihai, China), by means of an intramuscular injection ( $3 \mathrm{ml} / \mathrm{kg}$, body weight). Following anesthetization, the two sides of the femoral vein $(4 \mathrm{~cm})$ of the rabbits were exposed and separated from the surrounding tissue, taking care not to damage the femoral artery and nerve. Each end of the femoral vein was then completely ligated using 2-0 silk thread (Weihai Wego Medical Systems Co., Ltd; Fig. 1).

Delivery method. A total of 30 rabbits were randomly divided into three groups, each containing 10 rabbits: Control, model and Salvia. The Salvia group were treated with a daily intravenous injection of $2 \mathrm{~g} / \mathrm{kg} \mathrm{S}$. miltiorrhiza nto the ear vein prior to generation of the ligation model. The model and control groups received equal amounts of sodium chloride. Following one week of injections the rabbit ligation models were generated, according to the protocol described above. Each group of rabbits received the same treatment as mentioned until the end of the experiment. The response to stimuli (sensitivity to pain stimuli), activity levels and appetite in the rabbits were recorded. The rabbits were sacrificed by aeroembolism seven days after generation of the ligation model.

Specimen collection. Venous blood was collected in the normal and heparin tubes (Weihai Wego Medical Systems Co., Ltd), in order to measure SOD activities, MDA content, coagulation function (including prothrombin, activated partial prothrombin, fibrinogen and thrombin times) and blood rheological parameters (including whole blood viscosity, plasma viscosity and erythrocyte aggregation). Coagulation function was measured by CoaguChek ${ }^{\circledR}$ (Roche Diagnostics, Xinqin, China) and blood rheological parameters were measured using a Blood Rheology Analyzer (SA-9000; Lemon, Zhejing, China). These measurements were made prior to generation of the model, and three and seven days afterwards. At the end of the seven days the ligated femoral veins were placed in $10 \%$ formaldehyde solution, fixed for $48 \mathrm{~h}$ and paraffin-embedded. Serial sections $(4 \mu \mathrm{m})$ were collected for hematoxylin and eosin staining. Images of the venous wall and thrombosis were captured under a microscope (BX41; Olympus Corp., Tokyo, Japan). Low magnification (x10) was used to evaluate the vascular wall, while endothelial cells were observed at a higher magnification (x40).

Statistical analysis. The data were analyzed by Student's t-test and one-way analysis of variance to compare the differences between the groups, using SPSS version 13.0 software (SPSS Inc., Chicago, IL, USA). $\mathrm{P}<0.05$ was considered to indicate a statistically significant difference.

\section{Results}

Toxicity and quality of life. The response to stimuli, activity levels and appetite of the model and Salvia groups were similar to that of the control group. Furthermore, no fatal PE occurred in any of the rabbits.

Detection of coagulation function prior to generation of the ligation model. Following one week of S. miltiorrhiza treatment, the coagulation function of the Salvia group was not affected, except for prothrombin time (PT), which was significantly increased, as compared with the control group $(\mathrm{P}<0.05$; Fig. 2).

Measurements of blood rheological parameters and coagulation function, three days following generation of the ligation model. Following generation of the model, blood rheological parameters were measured, and whole blood viscosity $(1 / \mathrm{sec}$, 5/sec, 30/sec) and erythrocyte aggregation were shown to be significantly increased in the model group, as compared with the control group $(\mathrm{P}<0.05)$. These results suggest that ligation of the femoral vein may result in a hypercoagulable state. There was also a statistically significant increase in whole blood viscosity (1/sec, $5 / \mathrm{sec}, 30 / \mathrm{sec})$ in the Salvia group, as compared with the model group $(\mathrm{P}<0.05 ;$ Fig. 3). No significant differences were identified in the measurements of coagulation function, except for PT, which was increased in the Salvia group, as compared with the model group ( $\mathrm{P}<0.05$; Fig. 4).

Measurements of blood rheological parameters and coagulation function, seven days following generation of the ligation model. Seven days after generation of the model, blood rheological 
A

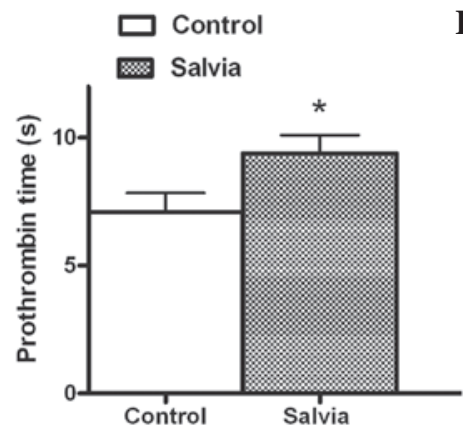

C

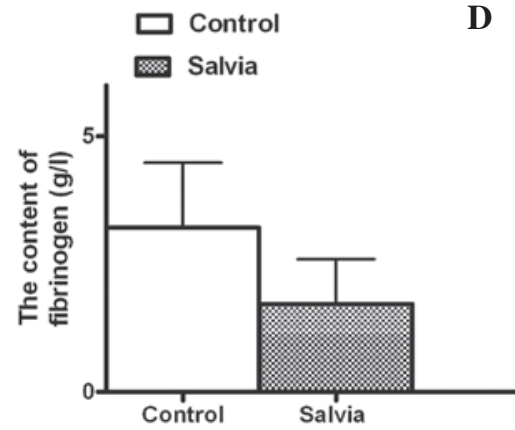

B

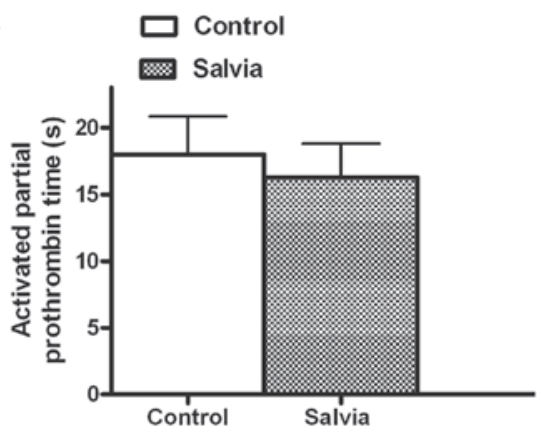

D

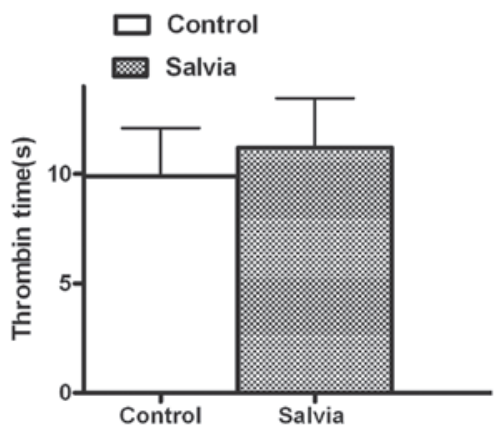

Figure 2. Effects after one week of intravenous infusion with Salvia miltiorrhiza. (A) Prothrombin time of the Salvia group was delayed compared with the control group ("P<0.05). (B-D) No statistically significant differences were identified in the other measurements of coagulation function, including activated partial prothrombin, fibrinogen and thrombin times $(\mathrm{P}>0.05)$.

A

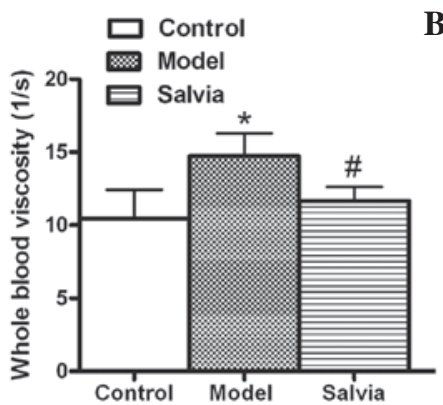

D

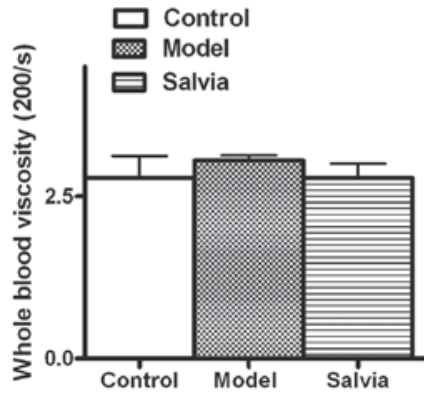

B

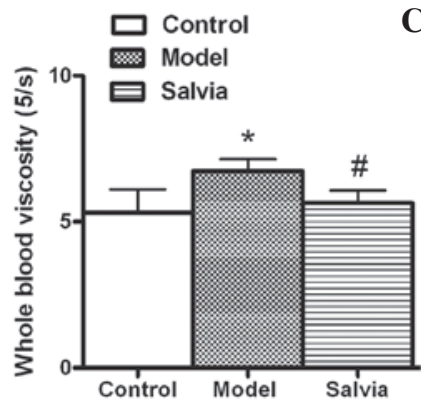

$\mathbf{E}$
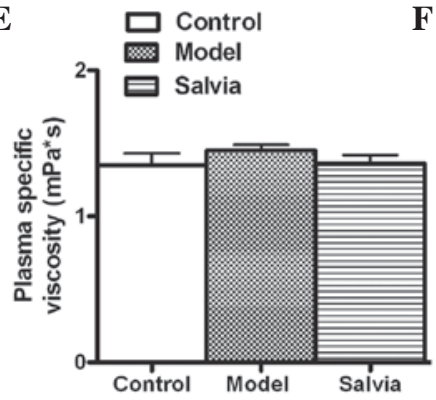

${ }^{*} \mathrm{P}<0.05$ vs. Control

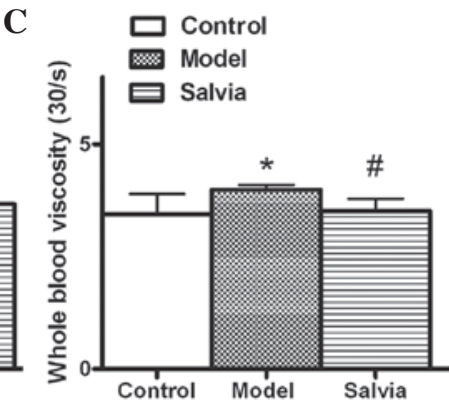

F

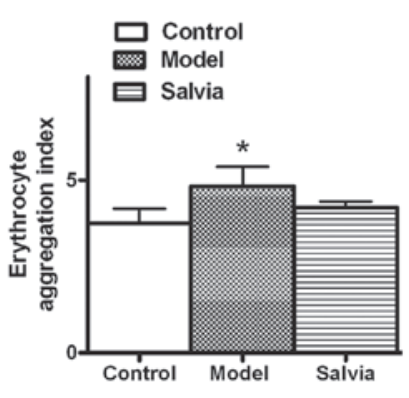

Figure 3. Measurements of blood rheological parameters were conducted three days after the generation of the ligation model. (A-C) There was a statistically significant difference in whole blood viscosity $(1 / \mathrm{sec}, 5 / \mathrm{sec}$ and $30 / \mathrm{sec})$ and $(\mathrm{F})$ erythrocyte aggregation index between the model and control groups $\left({ }^{*} \mathrm{P}<0.05\right)$. This suggested that ligation of the femoral vein results in a hypercoagulable state. (A-C) There was also a delay in the 1/sec, $5 / \mathrm{sec}$ and $30 / \mathrm{sec}$ whole blood viscosity in the Salvia group compared with the model group $\left({ }^{\#} \mathrm{P}<0.05\right)$. (D-F) No significant differences were identified in the other blood rheological parameters between the groups, including 200/sec whole blood viscosity, plasma specific viscosity and erythrocyte aggregation index $(\mathrm{P}>0.05)$.

parameters were measured, and whole blood viscosity $(1 / \mathrm{sec}$, $5 / \mathrm{sec}, 30 / \mathrm{sec}$ and $200 / \mathrm{sec}$ ) was shown to be significantly decreased in the Salvia group, as compared with the model group $(\mathrm{P}<0.05$; Fig. 5). There were no significant differences in the coagulation function between the Salvia and model groups (P>0.05; Fig. 6).
Antioxidative function. The present study evaluated the antioxidative function of S. miltiorrhiza, the results of which are presented in Fig. 7. The MDA content was significantly decreased $(\mathrm{P}<0.05)$, and the activities of SOD were notably increased $(\mathrm{P}<0.05)$ in the Salvia group compared with the 
A

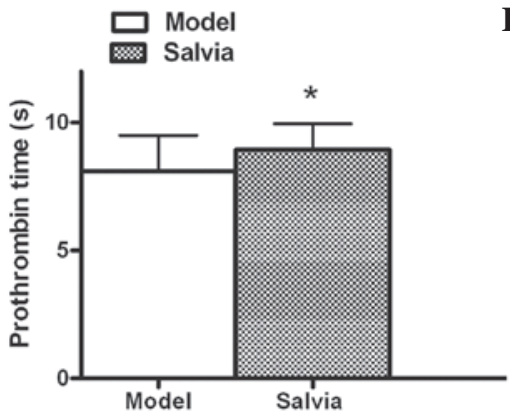

C

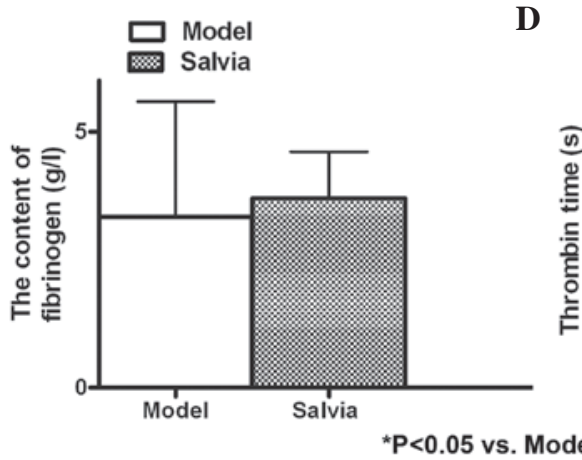

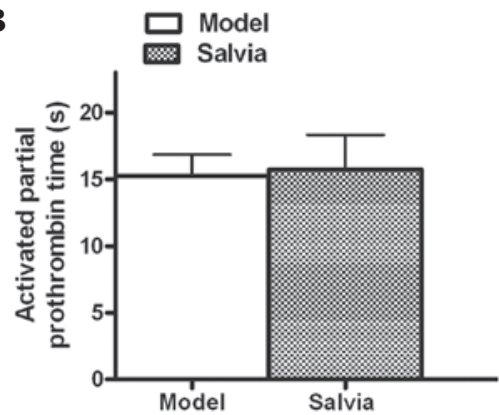

D

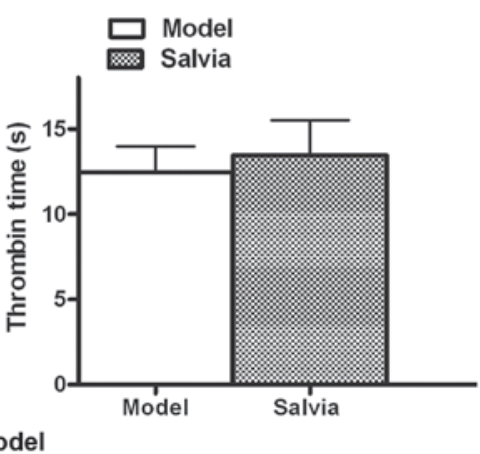

Figure 4. Effects three days after the generation of the ligation model. (A) Prothrombin time of the Salvia group was delayed compared with the model group $($ ( $\mathrm{P}<0.05)$. (B-D) No significant differences were identified in the other measurements of coagulation function between the groups, including activated partial prothrombin, fibrinogen and thrombin times $(\mathrm{P}>0.05)$.

A

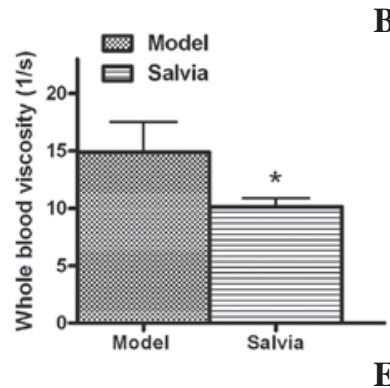

D

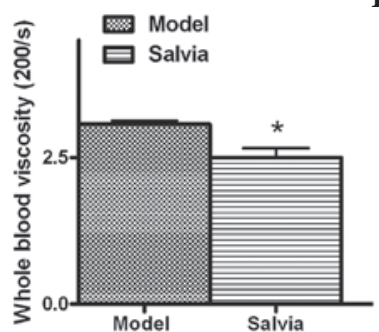

B

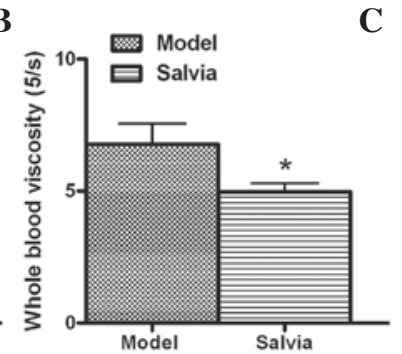

E

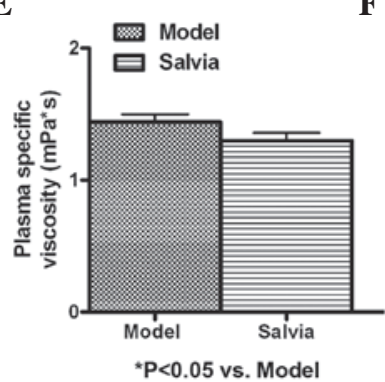

C

F
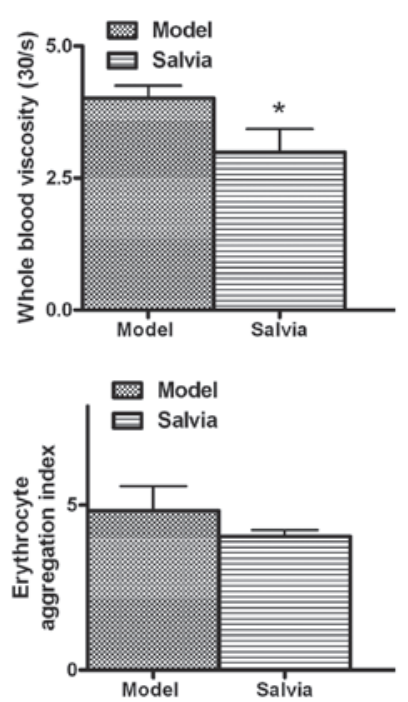

Figure 5. Effects seven days after the generation of the ligation model. (A-D) There was a significant increase in whole blood viscosity (1/s, 5/s, 30/s and 200/s) in the Salvia group compared with the model group $\left({ }^{*} \mathrm{P}<0.05\right)$. (E and F) No significant differences were identified in plasma-specific viscosity and erythrocyte aggregation index between the groups $(\mathrm{P}>0.05)$.

model group three days after generation of the model. The MDA content was higher on the seventh day $(8.00 \pm 1.96)$ in the model group compared with the third day $(5.63 \pm 1.77)$. However, in response to treatment with $S$. miltiorrhiza, MDA content was decreased $(\mathrm{P}<0.05)$, and SOD activities were increased $(\mathrm{P}<0.05)$ compared with the model group seven days after generation of the ligation model.

Histopathological observation. Following the sacrifice of the rabbits, the ligated femoral veins were harvested and images of the veins were captured under a microscope. The vascular wall was continuous in the control group, endothelial integrity was maintained and no shedding of endothelial cells was observed. The vascular wall of the model group exhibited discontinuous change, intimal irregularities, endothelial cell shedding, and thrombosis (mixed thrombus and red thrombus), which was attached to the vascular wall. The vascular wall of the Salvia group exhibited complete continuity, regular intima, some endothelial cell shedding and a small amount of intraluminal thrombosis, which was partly attached to the vascular wall (Fig. 8). 

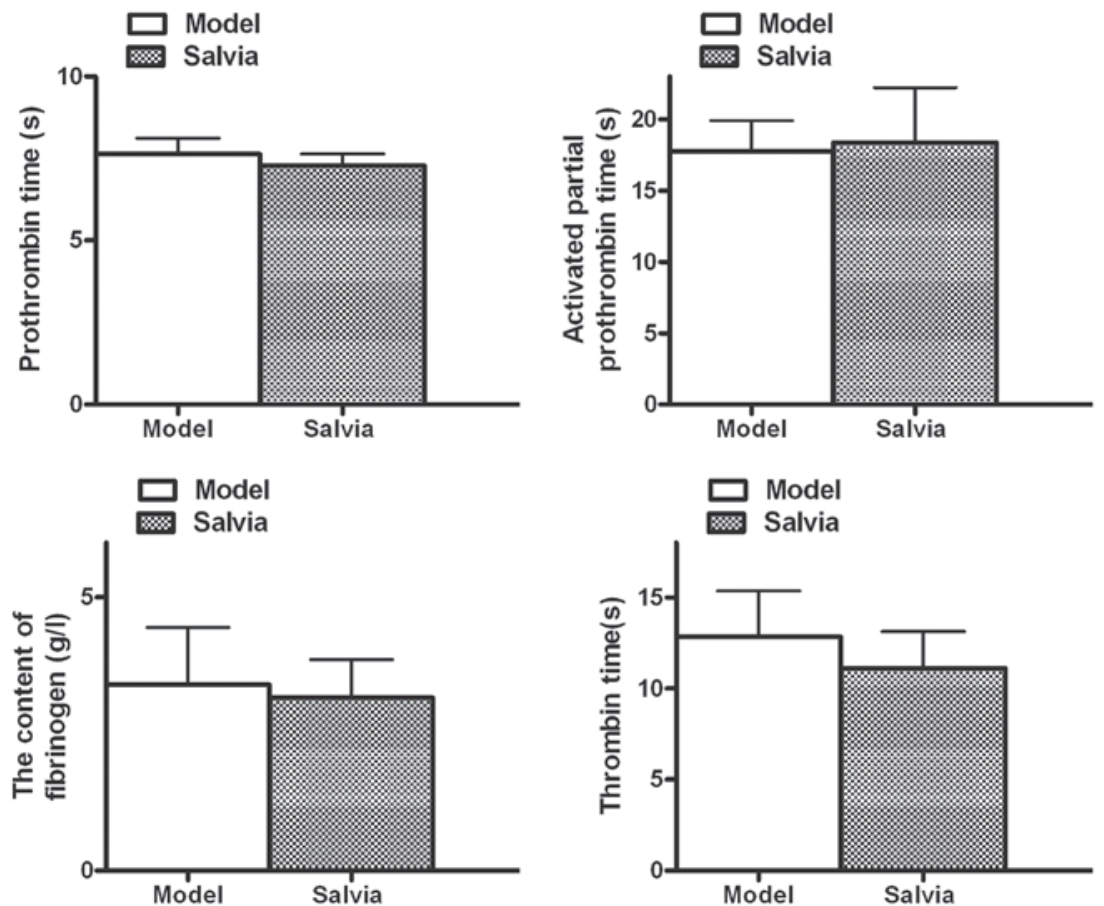

Figure 6. Seven days after the generation of the ligation model, no significant differences were identified in any of the measurements of coagulation function between the groups $(\mathrm{P}>0.05)$.

A
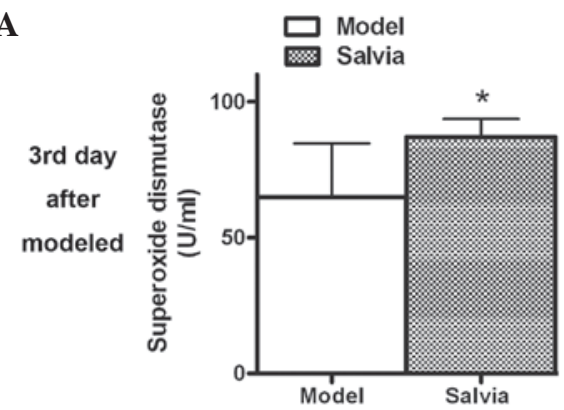

C

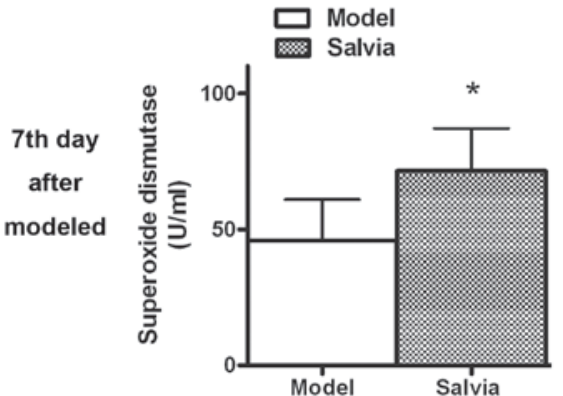

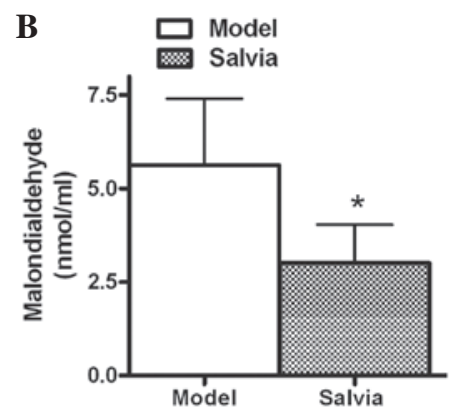

D $\square$ Model

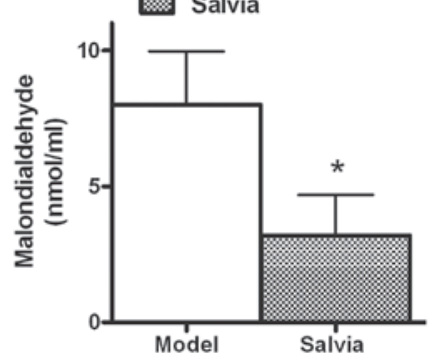

${ }^{\star} \mathrm{P}<0.05$ vs. Model

Figure 7. In the Salvia group, (A) activities of superoxide dismutase (SOD) were notably increased and the (B) malondialdehyde (MDA) content was significantly decreased compared with the model group three days after generation of the ligation model ("P<0.05). (C) MDA content was higher on the seventh day $(8.00 \pm 1.96)$ compared with the third day $(5.63 \pm 1.77)$ in the model group. (C and D) In response to treatment with $S$. miltiorrhiza, SOD activities increased, and MDA content decreased compared with the model group seven days after generation of the model $\left({ }^{*} \mathrm{P}<0.05\right)$.

\section{Discussion}

DVT is a well-known major public health problem, which represents a significant clinical and economic disease burden. Up to $21 \%$ of cases of DVT may lead to PE, which is a potentially life-threatening complication (12). The conventional treatment for acute DVT is immediate anticoagulation using low molecular-weight heparin, followed by a period (3-6 months) of treatment with oral anticoagulants $(13,14)$. This treatment aims to prevent thrombus propagation, and to reduce the risks of PE and DVT recurrence. However, anticoagulation therapy does not possess significant fibrinolytic activity, and patients with severe, extensive and proximal DVTs remain at high risk (15). In DVT treated with anticoagulants alone, it 
A

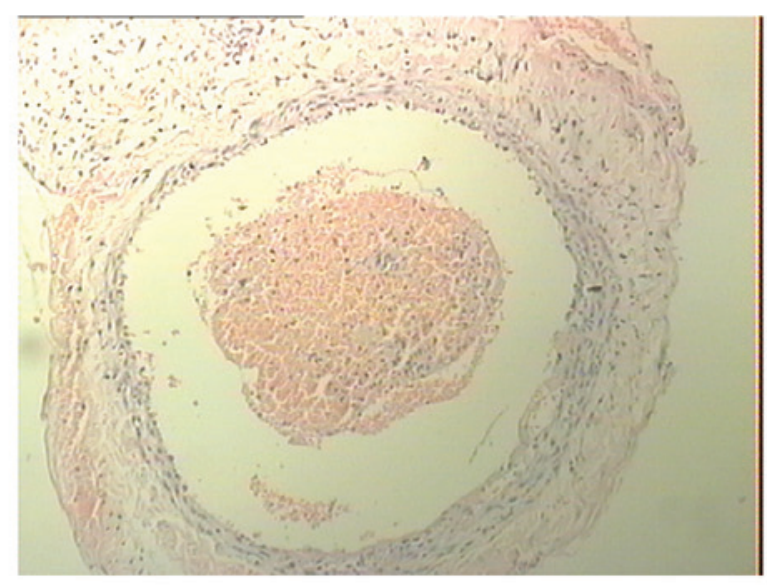

C

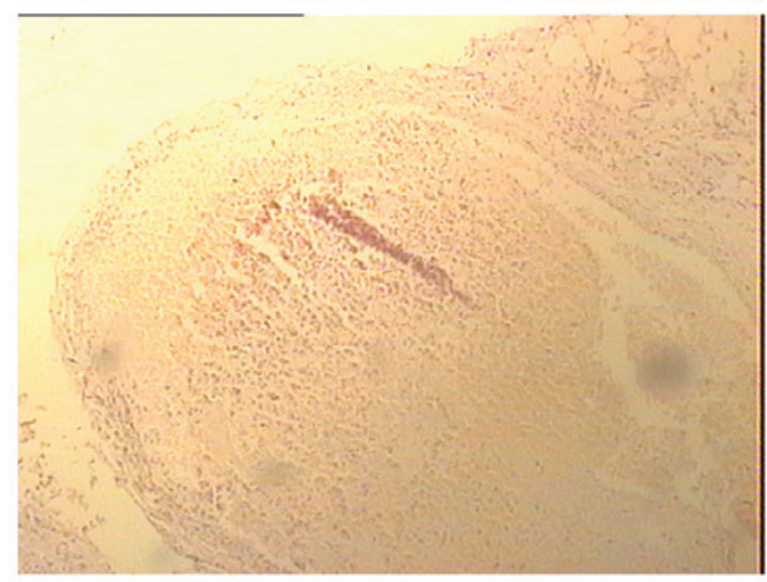

E

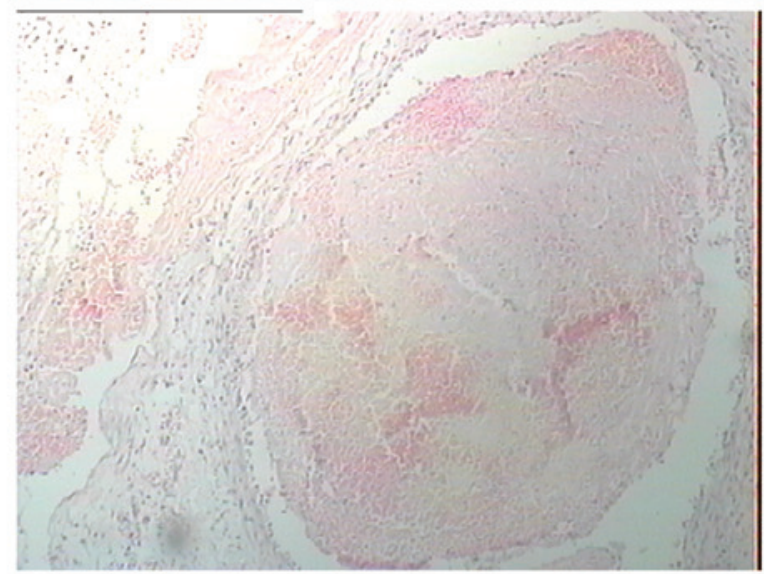

B

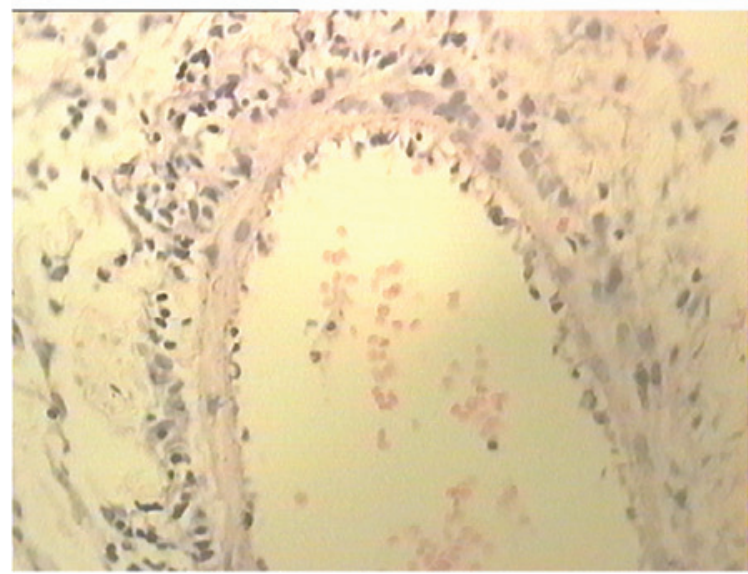

D

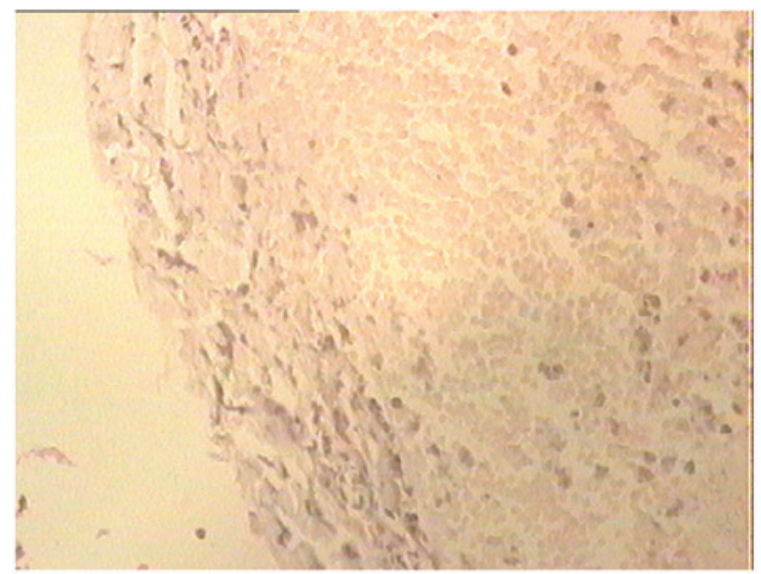

F

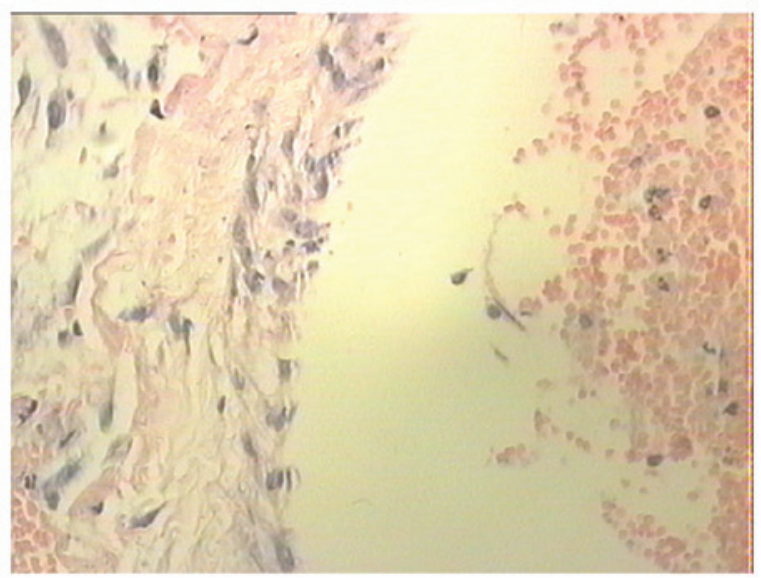

Figure 8. Images of the venous wall were captured under a microscope. (A and B) In the control group the vascular wall was continuous, endothelial integrity was maintained and no shedding of endothelial cells was observed. (C and D) In the model group the vascular wall showed discontinuous change, intimal irregularities, endothelial cell shedding and thrombosis (mixed and red thrombi), which was attached to the vein vascular wall. (E and F) In the Salvia group the vascular wall exhibited complete continuity, regular intima, some endothelial cell shedding and a small amount of intraluminal thrombosis, which was partly attached to the vein vascular wall. (A, C and E) Hematoxylin and eosin (H\&E); magnification, x10. (B, D and F) H\&E; magnification, x40.

has been shown that early spontaneous clot lysis frequently results in preservation of valvular function, which may help to reduce post-thrombotic morbidity (16). The present study chose to administer an intravenous injection of $S$. miltiorrhiza one week prior to the generation of a ligation model in rabbits.

S. miltiorrhiza is a common traditional Chinese medicine used for improving body function, which is capable of promoting circulation and improving blood flow. In addition, it has been used for the treatment of cardiovascular diseases, including coronary heart disease, hyperlipidemia and cerebrovascular disease $(17,18)$. S. miltiorrhiza has also been widely used in the
United States (10). When platelets are challenged with outside stimuli to vascular endothelial cells they increase the expression of the adhesion molecule CD31, which may adhere to vascular endothelial cells, resulting in the formation of a soft thrombus (19). Furthermore, the formation of hard thrombi is initiated by the enhanced adhesion of platelets, and the conversion of fibrinogen to fibrin (19). Numerous studies have been conducted regarding the effects of S. miltiorrhiza on platelet aggregation. The inhibitory effects of $S$. miltiorrhiza have been suggested to be associated with numerous events, including the inhibition of $\mathrm{Ca}^{2+}$ influx in platelets, an increase in the number 
of fibroblasts in the $G_{0} / G_{1}$ phase and the attenuation of collagen secretion (20). The present study demonstrated that treatment with S. miltiorrhiza one week after generation of a ligation model, did not affect the majority of measurements of coagulation function, except for PT, which can be used to evaluate the action of five different clotting factors (I, II, V, VII, and X). Blood that takes a long time to clot in a PT test has previously been shown to be an indicator of treatment using warfarin (21). In the present study the PT was significantly increased in the Salvia group on the third day, but not on the seventh day, after generation of the model.

Endothelium secretes factors that control vascular relaxation and contraction, thrombogenesis and fibrinolysis, and platelet activation and inhibition (22). Therefore, maintenance of the functional integrity of endothelium is critical for preservation of blood flow, and the prevention of thrombosis (23). A balance between growth and death of endothelial cells is important for the integrity of the vascular endothelium. An appropriate growth response of endothelial cells helps maintain the integrity of the endothelium, and prevent the development of atherosclerosis (24). The results of the present study indicated that $S$. miltiorrhiza may protect vascular endothelial cells in vivo. Previous studies have also demonstrated the protective functions of $S$. miltiorrhiza on human vascular endothelial cells in vitro (25-27).

Oxidative stress is the imbalance between the levels of antioxidants and the production of oxygen-derived species (28). In the present study $S$. miltiorrhiza exhibited antioxidative functions in the vein ligation model. Chan et al (29) investigated the effects of S. miltiorrhiza on the pharmacodynamics and pharmacokinetics of warfarin in rats. S. miltiorrhiza was shown to potentiate the anticoagulant action of warfarin, by increasing the absorption rate constant, the area under the plasma concentration time curve, and the maximum concentration and half-life of warfarin. In addition, S. miltiorrhiza decreased the clearance and the apparent volume of distribution of warfarin. The present study did not investigate the effects of warfarin, or the effects of a combined treatment of S. miltiorrhiza with warfarin.

In conclusion, previous studies indicated that S. miltiorrhiza promoted circulation and improved blood flow in the treatment of cardiovascular diseases, while the present study revealed that S. miltiorrhiza exhibited antioxidative and protective effects on vascular endothelial cells. The results of the present in vivo study demonstrated that $S$. miltiorrhiza can decrease blood rheological parameters. S. miltiorrhiza was also shown to exhibit antioxidative and protective effects on vascular endothelial cells. These results suggest that $S$. miltiorrhiza may have potential applications for the treatment of DVT.

\section{Acknowledgements}

The present study was supported by the Hubei Provincial Department of Education (grant no. Q20132108).

\section{References}

1. Anderson FA Jr., Wheeler HB, Goldberg RJ, et al: A population-based perspective of the hospital incidence and case-fatality rates of deep vein thrombosis and pulmonary embolism. The Worcester DVT Study. Arch Intern Med 151: 933-938, 1991.
2. Brown HL and Hiett AK: Deep vein thrombosis and pulmonary embolism in pregnancy: diagnosis, complications, and management. Clin Obstet Gynecol 53: 345-359, 2010.

3. Jaff MR, McMurtry MS, Archer SL, et al: Management of massive and submassive pulmonary embolism, iliofemoral deep vein thrombosis, and chronic thromboembolic pulmonary hypertension: a scientific statement from the American Heart Association. Circulation 123: 1788-1830, 2011.

4. Hardwick ME, Pulido PA and Colwell CW, Jr.: A mobile compression device compared with low-molecular-weight heparin for prevention of venous thromboembolism in total hip arthroplasty. Orthop Nurs 30: 312-316, 2011.

5. Orken DN, Kenangil G, Ozkurt H, et al: Prevention of deep venous thrombosis and pulmonary embolism in patients with acute intracerebral hemorrhage. Neurologist 15: 329-331, 2009.

6. Lawless RA and Dangleben DA: Caval agenesis with a hypoplastic left kidney in a patient with trauma on warfarin for deep vein thrombosis. Vasc Endovascular Surg 46: 75-76, 2012.

7. Battistelli S, Genovese A and Gori T: Heparin-induced thrombocytopenia in surgical patients. Am J Surg 199: 43-51, 2010.

8. Scarpa M, Pilon F, Pengo V, et al: Deep venous thrombosis after surgery for inflammatory bowel disease: is standard dose low molecular weight heparin prophylaxis enough? World J Surg 34: 1629-1636, 2010

9. Yu S, Zhong B, Zheng M, Xiao F, Dong Z and Zhang H: The quality of randomized controlled trials on DanShen in the treatment of ischemic vascular disease. J Altern Complement Med 15: 557-565, 2009.

10. Cheng TO: Cardiovascular effects of Danshen. Int J Cardiol 121: 9-22, 2007.

11. Ho JH and Hong CY: Salvianolic acids: small compounds with multiple mechanisms for cardiovascular protection. J Biomed Sci 18: 30, 2011.

12. Iverson RE and Gomez JL: Deep venous thrombosis: prevention and management. Clin Plast Surg 40: 389-398, 2013.

13. Wang YP, Zhang XQ, Yu WN, et al: Endovascular treatment of acute proximal deep venous thrombosis secondary to iliac vein compression syndrome: a novel technique for thrombus removal. Chin Med J (Engl) 126: 3184-3186, 2013.

14. Deep venous thrombosis and pulmonary embolism. Part 1. Initial treatment: usually a low-molecular-weight heparin. Prescrire Int 22: 99-101, 103-104, 2013.

15. Cho ES, Chung JJ, Kim S, Kim JH, Yu JS and Yoon CS: CT venography for deep vein thrombosis using a low tube voltage (100 $\mathrm{kVp}$ ) setting could increase venous enhancement and reduce the amount of administered iodine. Korean J Radiol 14: 183-193, 2013.

16. Liew A and Douketis J: Initial and long-term treatment of deep venous thrombosis: recent clinical trials and their impact on patient management. Expert Opin Pharmacother 14: 385-396, 2013.

17. Wen XD, Wang CZ, Yu C, et al: Salvia miltiorrhiza (dan shen) significantly ameliorates colon inflammation in dextran sulfate sodium induced colitis. Am J Chin Med 41: 1097-1108, 2013.

18. Zhang JP, Zhang YY, Zhang Y, et al: Salvia miltiorrhiza (Danshen) injection ameliorates iron overload-induced cardiac damage in mice. Planta Med 79: 744-752, 2013.

19. Gurbel PA, Serebruany VL, Shustov AR, et al: Increased baseline levels of platelet P-selectin, and platelet-endothelial cell adhesion molecule-1 in patients with acute myocardial infarction as predictors of unsuccessful thrombolysis. Coron Artery Dis 9: 451-456, 1998 .

20. Han JY, Fan JY, Horie Y, et al: Ameliorating effects of compounds derived from Salvia miltiorrhiza root extract on microcirculatory disturbance and target organ injury by ischemia and reperfusion. Pharmacol Ther 117: 280-295, 2008.

21. Arbring K, Uppugunduri S and Lindahl TL: Comparison of prothrombin time (INR) results and main characteristics of patients on warfarin treatment in primary health care centers and anticoagulation clinics. BMC Health Serv Res 13: 85, 2013.

22. Axtell AL1, Gomari FA and Cooke JP: Assessing endothelial vasodilator function with the Endo-PAT 2000. J Vis Exp 15: 2167, 2010.

23. Luscher TF and Barton M: Biology of the endothelium. Clin Cardiol 20 : II-3-10, 1997.

24. Ling S, Nheu L, Dai A, Guo Z and Komesaroff P: Effects of four medicinal herbs on human vascular endothelial cells in culture. Int J Cardiol 128: 350-358, 2008.

25. Zhou Z, Wang SQ, Liu Y and Miao AD: Cryptotanshinone inhibits endothelin-1 expression and stimulates nitric oxide production in human vascular endothelial cells. Biochim Biophys Acta 1760: 1-9, 2006. 
26. Chan P, Chen YC, Lin LJ, Cheng TH, Anzai K, Chen YH, Liu ZM, Lin JG and Hong HJ: Tanshinone IIA attenuates $\mathrm{H}_{2} \mathrm{O}_{2}$-induced injury in human umbilical vein endothelial cells. Am J Chin Med 40: 1307-1319, 2012.

27. Zhou ZW, Xie XL, Zhou SF and Li CG: Mechanism of reversal of high glucose-induced endothelial nitric oxide synthase uncoupling by tanshinone IIA in human endothelial cell line EA.hy926. Eur J Pharmacol 697: 97-105, 2012

28. Møller P, Danielsen PH, Karottki DG, Jantzen K, Roursgaard M, Klingberg H, Jensen DM, Christophersen DV, Hemmingsen JG, Cao Y and Loft S: Oxidative stress and inflammation generated DNA damage by exposure to air pollution particles. Mutat Res Rev Mutat Res 762C: 133-166, 2014.
29. Chan K, Lo AC, Yeung JH and Woo KS: The effects of Danshen (Salvia miltiorrhiza) on warfarin pharmacodynamics and pharmacokinetics of warfarin enantiomers in rats. J Pharm Pharmacol 47: 402-406, 1995. 